\title{
Least Biased Target Selection in Probabilistic Atlas Construction
}

\author{
Hyunjin Park ${ }^{1}$, Peyton H. Bland ${ }^{1}$, Alfred O. Hero III $^{2}$, and Charles R. Meyer ${ }^{1}$ \\ ${ }^{1}$ Department of Radiology, University of Michigan, Ann Arbor, MI, USA \\ \{hyunjinp, bland, hero, cmeyer\} @umich.edu \\ ${ }^{2}$ Department of Electric Engineering and Computer Science, \\ University of Michigan, Ann Arbor, MI, USA
}

\begin{abstract}
Probabilistic atlas has broad applications in medical image segmentation and registration. The most common problem building a probabilistic atlas is picking a target image upon which to map the rest of the training images. Here we present a method to choose a target image that is the closest to the mean geometry of the population under consideration as determined by bending energy. Our approach is based on forming a distance matrix based on bending energies of all pair-wise registrations and performing multidimensional scaling (MDS) on the distance matrix.
\end{abstract}

\section{Introduction}

The probabilistic atlas has been widely used to bring useful prior information to segmentation and registration tasks of human organs, especially for brain. In the segmentation task, atlas information may be used as prior information in the Bayesian formulation [1]. Atlas information guides segmentation algorithms where there is little grayscale value information available.

A common approach to building an atlas is to first pick a target image and map other images onto that target so that statistical processing can be done in the same spatial frame. Methods for registration (i.e., mapping) in terms of degrees of freedom (DOF) and geometric interpolant have to be the same for all registration tasks to ensure consistent construction and use of the atlas. The resulting atlas is inherently biased towards the chosen target image. If the target image happens to be an extreme case of the population, then the atlas created does not reflect the population correctly. In that case, bringing an arbitrary image to the atlas space, i.e., registering a test image onto the atlas, maybe difficult since the geometric distance the test image has to travel to reach the atlas has been increased compared to the case of reaching an atlas which resides at the mean geometry of the population. The whole process of mapping other images onto the target may be repeated with the target replaced with an average image from the previous registrations until the average image converges [2]. In this case, the bias towards the initially picked target image may be reduced.

Studholme et al. proposed a method to jointly register all images simultaneously to a target space that is very close to the mean geometry to reduce the bias of the target space [3]. It employs a cost function encouraging mean displacement field from the target onto other images to be zero while minimizing the joint entropy of all images. 
All displacement fields to other images have to be known to compute the mean displacement field, thus the method requires registration of all images simultaneously increasing the optimization space tremendously. There are nontrivial issues in how to compute the high dimensional probability density function needed to evaluate the joint entropy. Joshi et al. used an atlas construction independent of choosing a specific target image [4]. They first construct an atlas by mapping other images onto a target image and performing statistical processing. After the atlas is constructed on the target image space, the atlas is warped onto a space where there is less bias towards the rest of the images. As a result they can choose any target image and arrive at the same atlas space since the atlas calculated on a specific target image space is always going to be warped onto another space where there is less bias. The above approach needs to satisfy certain constraints that will be discussed in section 4. Marsland et al. proposed to construct the atlas on a target image that is the closest to the mean geometry [10]. They choose the target image such that sum of distances from the target image to the rest of the images is minimized. Our work shares a similar approach to Marsland's paper. Improvements resulting from using our approach will be discussed.

Here we present a method to choose a target image that is the closest to the mean geometry. We acknowledge the existing work on unbiased atlas construction and provide an alternative method based on novel statistical machinery. Our approach is based on forming a distance matrix based on bending energies of all pair-wise registrations and using multidimensional scaling (MDS) on the distance matrix to find the closest target.

\section{Methods}

In this paper we choose the most common atlas construction method, mapping other images onto a chosen target image and performing statistical processing on the target image space. Our contribution is how to choose a target image that is the least biased considering all the other images.

\subsection{Pair-Wise Registration}

The task of mapping one image onto the other image is carried out by registration. Registration has been well discussed [5]. In short, two main components need to be determined for any registration method: the similarity measure which measures degree of alignment between images, and the geometric interpolant which defines the geometric transform. We choose mutual information (MI) as the similarity measure and thin-plate splines (TPS) as the geometric interpolant [6]. A simple histogram with fixed bin width is used to calculate the probability mass function of grayscale value distributions to compute MI. The process of registration can be formulated as maximizing the chosen similarity measure (i.e., MI) under a hypothetical geometric transform.

\subsection{Distance Measure}

Registration between two images yields a geometric transform optimized to maximize a certain cost function (e.g., MI). The geometric distance, hereafter called distance, 
between two images is often measured by the roughness of the geometric transform. In addition, having zero distance for a simple affine transform is desirable. We define distance between two images as the sum of squared second partial derivatives of the geometric transform,

$$
d^{2}=\iint\left(\frac{\partial^{2} f_{x}}{\partial x^{2}}\right)^{2}+2\left(\frac{\partial^{2} f_{x}}{\partial x \partial y}\right)^{2}+\left(\frac{\partial^{2} f_{x}}{\partial y^{2}}\right)^{2}+\left(\frac{\partial^{2} f_{y}}{\partial x^{2}}\right)^{2}+2\left(\frac{\partial^{2} f_{y}}{\partial x \partial y}\right)^{2}+\left(\frac{\partial^{2} f_{y}}{\partial y^{2}}\right)^{2} d x d y
$$

$f_{x}$; displacement in $\mathrm{x} f_{y}$; displacement in $\mathrm{y}$.

Above formulation is for 2D and can be easily extended for 3D. This distance is often called the bending energy. Analytic formula for calculating bending energy is available for TPS [7]. For other geometric transforms, the bending energy may need to be calculated numerically. The defined distance is not strictly a metric since the distance between two different images can be zero if the two images can be registered by an affine transform.

\subsection{Multidimensional Scaling (MDS)}

Multidimensional scaling (MDS) is a technique to produce relative positional locations from a collection of pair-wise distances [8]. For example, given pair-wise Euclidean distances between North American cities, MDS will yield a map of relative locations (i.e., up to an arbitrary rotate-translate transform) of those cities. For $\mathrm{N}$ cities, $\mathrm{N}(\mathrm{N}-1) / 2$ (i.e., $\mathrm{N}$ choose 2) pair-wise distances are needed. The distances used in MDS need not be metric; non-metric distances (e.g., ranking) can be used. Thus, our distance defined in section 2.2 is valid in MDS settings. Given a set of distances in the distance matrix $D$, whose element $d_{i j}$ refers to the distance between objects $i$ and $\mathrm{j}$, MDS outputs a set of coordinates in a user specified dimension that reproduces the distance matrix best in the least square fashion. The dimension of MDS output should be based on the eigen structure of the distance matrix. The output coordinates are in the standard Euclidean space of the user supplied dimension.

\subsection{Target Selection Based on MDS}

An ideal target image is the one that resides at the mean geometry of the population. For the ideal target image, the sum of distances to other images from the atlas space (i.e., target space) is minimized. For a target image space that is far away from the mean geometry, the sum of distances to other images will be greater. Often there may not be an image at the mean geometry thus the best approach in picking a target image, which yields the minimum distance to other images, is to choose the image that is the closest to the mean geometry. The described approach works only if we know all the relative locations of images of the population. MDS identifies all the relative locations of the images from the distance matrix. Here the elements of the distance matrix are determined by the distances of pair-wise registrations. In summary, we select the target image which is the closest to the mean geometry with the aid of information of relative locations provided by MDS. MDS in turn requires a distance matrix whose elements are calculated from pair-wise registrations. The following is the procedure for $\mathrm{N}$ images, 
1. Perform $N(N-1) / 2$ pair-wise registrations

2. Calculate bending energies from the registrations

3. Form distance matrix D

4. Apply MDS and find relative locations of images

5. Calculate mean location of the images

6. Choose target image that is the closest to the mean.

Once the best (i.e., least biased) target is selected, all other images can be mapped on to the chosen target, this is trivial since all pair-wise registrations have been computed previously to fill the distance matrix.

\subsection{Distance Matrix}

The distance matrix is either symmetric or asymmetric. For a symmetric distance matrix, distance between object $i$ and $j$ is order independent. In atlas construction, it implies that the distance between image $i$ as the reference image and image $j$ as the floating image is the same as the distance between images $i$ and $j$ switching the role of the reference image and the floating image. In practice with TPS based registrations, switching the order of images in the registration task may yield a different geometric transform thus it may yield a different distance value, but the discrepancy in distance value is quite small provided that the degrees of freedom (DOF) of TPS is high enough. Christensen et al. proposed a registration method where both forward and inverse transform are estimated such that switching the order of reference and floating images has little effect [11]. In this case, symmetric distance matrix is ensured. Even for an asymmetric distance matrix, distance matrix can be made symmetric by using the average value of $\mathrm{d}_{\mathrm{ij}}$ and $\mathrm{d}_{\mathrm{ji}}$. Here we assume a symmetric distance matrix thus we only calculate upper half of the distance matrix and replicate the lower half. The diagonal elements of the distance matrix are zero by definition.

\section{Experiments}

Synthetic experiments are carried out in 2D to show the feasibility of our approach. A synthetic MRI slice, 256x256 dimension and $1 \times 1 \mathrm{~mm}^{2}$ resolution, is obtained from BRAINWEB simulation [9]. It is deformed in a known way using a $6 \times 6$ grid of Bsplines resulting in 50 deformed images. Deformations are applied by randomly choosing a knot and displacing the knot by the amount determined by zero mean Gaussian of variance 100 pixels in both $\mathrm{x}$ and $\mathrm{y}$ direction. After the image's geometry is deformed, a zero mean Gaussian noise of variance 16 is added to the image's grayscale values. Six images of the known 50 deformed images are shown in Figure 1. The atlas is constructed with these 50 deformed images. Geometric distances from the original undeformed image (i.e., BRAINWEB slice) to all 50 images are calculated given all the known synthetic deformations and are shown in Table 1. With these distances, ground truth on what is the best target image can be established. The best target image is the image that is the closest to the original image (i.e., the least distance from the original image). In addition, the quality of all potential target images can be rank ordered according to the distance from the original image. 


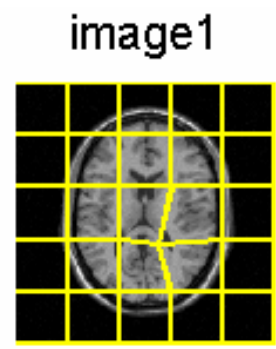

image4

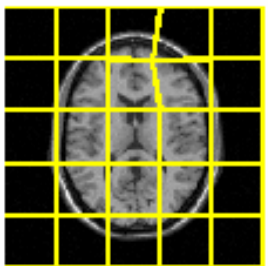

image2

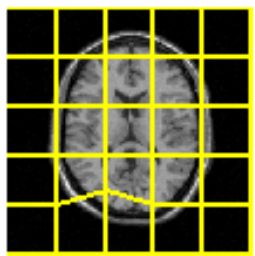

image8

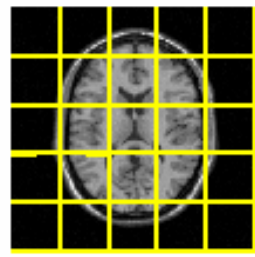

image3

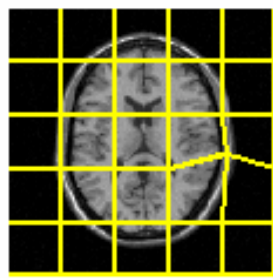

image15

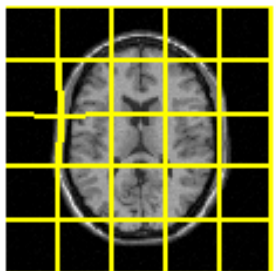

Fig. 1. Six images of the known 50 deformed images. Grid lines show the applied B-spline deformations. Image 8 and 15 have very small deformations compared to other images shown.

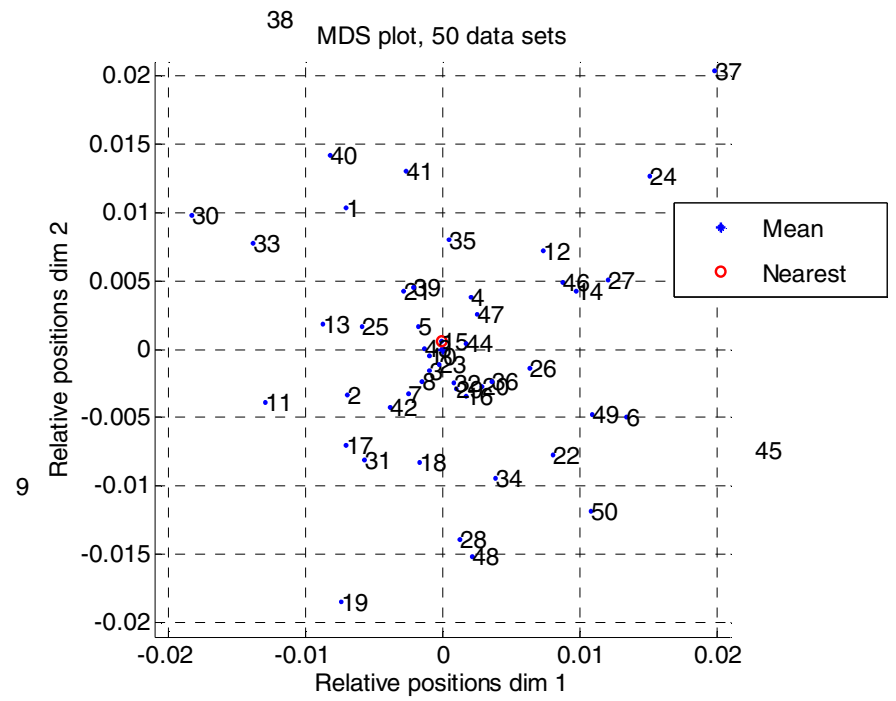

Fig. 2. Relative locations of 50 images by MDS. Mean location is at $(0,0,0,0)$ and the closest image to mean is determined to be image 15 . Only a 2 dimensional plot (out of 4 ) is given here for space constraints. Mean is marked with ' + ' and the nearest image to the mean is marked with ' $\mathrm{o}$ '.

Pair-wise registrations of the 50 images are performed using 25 uniformly spread control points. There are 1225 (i.e., 50 choose 2) pair-wise registrations required to fill up the symmetric 50x50 distance matrix. MDS is performed with 4 dimensions. 
Table 1. Distances from the original undeformed image. Geometric distances are sorted ascendingly. Images with small distances are desirable as the target image. Image 8 is the most desirable target image and image 9 is the least desirable target image.

\begin{tabular}{|c|c|c|c|c|c|c|c|c|c|}
\hline Distance & 0.0046 & 0.0055 & 0.0121 & 0.0152 & 0.0203 & 0.0209 & 0.0251 & 0.0321 & 0.0325 \\
\hline Image \# & 8 & 10 & 29 & 15 & 32 & 44 & 5 & 36 & 47 \\
\hline Distance & 0.0363 & 0.0598 & 0.0601 & 0.0777 & 0.0900 & 0.1170 & 0.1276 & 0.1350 & 0.1381 \\
\hline Image \# & 16 & 20 & 43 & 4 & 21 & 46 & 42 & 35 & 17 \\
\hline Distance & 0.1409 & 0.1483 & 0.1499 & 0.2146 & 0.2182 & 0.2279 & 0.2288 & 0.2322 & 0.2416 \\
\hline Image \# & 26 & 18 & 31 & 13 & 14 & 1 & 34 & 11 & 49 \\
\hline Distance & 0.2532 & 0.2581 & 0.2592 & 0.2785 & 0.2802 & 0.2881 & 0.2909 & 0.3289 & 0.3454 \\
\hline Image \# & 41 & 2 & 7 & 23 & 3 & 25 & 6 & 48 & 27 \\
\hline Distance & 0.3611 & 0.3660 & 0.3734 & 0.3860 & 0.4176 & 0.4704 & 0.4805 & 0.5009 & 0.5158 \\
\hline Image \# & 40 & 19 & 50 & 12 & 39 & 24 & 22 & 28 & 33 \\
\hline Distance & 0.5802 & 0.7047 & 0.8666 & 1.0827 & 1.3786 & & & & \\
\hline Image \# & 45 & 30 & 37 & 38 & 9 & & & & \\
\hline
\end{tabular}

Table 2. MDS results. Image number is sorted by the distance from the location of mean geometry. Distances are sorted ascendingly. The order of image number is very similar to the order of image number in Table 1. RMS (root mean squared) error between the order of images by MDS and order of images of the ground truth is computed on the bottom row.

\begin{tabular}{|c|c|c|c|c|c|c|c|c|c|}
\hline Order & 1 & 2 & 3 & 4 & 5 & 6 & 7 & 8 & 9 \\
\hline Image \# & 15 & 8 & 5 & 32 & 44 & 29 & 10 & 43 & 47 \\
\hline Order & 10 & 11 & 12 & 13 & 14 & 15 & 16 & 17 & 18 \\
\hline Image \# & 36 & 16 & 4 & 20 & 21 & 26 & 42 & 35 & 46 \\
\hline Order & 19 & 20 & 21 & 22 & 23 & 24 & 25 & 26 & 27 \\
\hline Image \# & 18 & 17 & 34 & 1 & 31 & 14 & 7 & 3 & 2 \\
\hline Order & 28 & 29 & 30 & 31 & 32 & 33 & 34 & 35 & 36 \\
\hline Image \# & 13 & 25 & 11 & 49 & 23 & 41 & 27 & 6 & 12 \\
\hline Order & 37 & 38 & 39 & 40 & 41 & 42 & 43 & 44 & 45 \\
\hline Image \# & 40 & 50 & 19 & 48 & 33 & 39 & 28 & 24 & 22 \\
\hline Order & 46 & 47 & 48 & 49 & 50 & Error & & & \\
\hline Image \# & 45 & 30 & 38 & 37 & 9 & 0.3970 & & & \\
\hline
\end{tabular}

The dimension is determined by observing the eigenvalue trend (i.e., abrupt drop in eigenvalues) of the distance matrix. Output of the MDS is 50 coordinates in 4 dimensions representing the 50 images in the Euclidean space. Two dimensional projections of these coordinates are shown in Figure 2. The location of mean geometry is calculated by taking the arithmetic mean of 50 coordinates, which is set to be the origin (i.e., $(0,0,0,0))$. The image whose coordinate is the closest to the mean geometry is chosen to be the best target image. Distances from the mean geometry (i.e., origin) to the images (i.e., MDS coordinates) are sorted in Table 2 starting from the closest image to the furthest image. The best target image (i.e., the first image in Table 2) is image 15 while the ground truth (i.e., Table 1) indicates image 8 to be the best target. Image 8 and 15 have bending energies 0.0046 and 0.0152 respectively according to Table 1. The difference of bending energies between image 8 and 15, 
0.0106 , is relatively small compared to the possible variation of bending energies from 0.0046 to 1.3786 (i.e., bending energies of image 8 and 9). Thus our selected target is reasonably close to the mean geometry if not the closest target. Moreover comparison of order of images in Table 1 and 2 indicates that MDS results, order of images in Table 2, are very similar to the ground truth's order of images in Table 1. We are able to replicate the order of images reasonably well from MDS results, not just the closest image to the mean geometry. In fact, the root mean squared error between the rank orders of images of Tables 1 and 2 is computed to be 0.3970. MDS coordinates are tested for multi-variate normal distribution and satisfy 4 dimensional normality test with $\mathrm{p}=1$ and alpha $=0.05$. It implies that the origin (i.e., $(0,0,0,0))$ represents the undeformed image and that the distribution is not skewed.

\section{Discussions and Summary}

Our target selection method based on MDS enables us to choose a target that is very close to the mean geometry. One potential reason for not arriving at the closest target may come from inaccuracies in the pair-wise registration processes. If the registration process is not accurate, then the distance matrix contains inaccurate elements. Thus, MDS results may be affected. Our approach is independent of the choice of the pairwise registration methods. User can choose any reasonable combination of similarity measure and geometric interpolant, not just MI and Thin-plate splines. Relative locations of the images can be found as well as the closest image from the mean geometry.

Our method is potentially faster than the iterative atlas construction method if the iterative method needs many iterations (i.e., at least N/2 iterations) to converge [2]. Our approach requires $\mathrm{N}(\mathrm{N}-1) / 2$ pair-wise registrations and the iterative construction method requires $(\mathrm{N}-1) \mathrm{x}$ (\# of iterations) pair-wise registrations for $\mathrm{N}$ images. Joshi's approach requires only $\mathrm{N}-1$ pair-wise registrations and is independent of choosing a target image [4]. It assumes that the geometric transform has certain "small deformation" properties and is sensitive to pair-wise registration results requiring displacement fields to be accurate (i.e., almost no registration error). Our approach and Marsland's approach share a common theme, i.e., finding a target image that is the closest to the mean geometry [10]. Their method tries to minimize not only the sum of distances to all other image but also the sum of similarity measure between the target and other images. They start with an initial guess of the target image and try to update the target image if the sum of distances decreases and the sum of MI increases. We believe target should be chosen solely on distances. For example, if the ideal target image at the mean geometry happens to be noisy, under Marsland's approach it will never be selected as the target since choosing the ideal target will surely decrease the sum of MI. Their method is tied to a specific geometric interpolant, clamped-plate spline, while our approach can be applied to any geometric interpolant.

We have shown a method to choose the target image that is very close to the mean geometry. It is based on information of relative locations provided by MDS. MDS requires a distance matrix whose elements are calculated from pair-wise registrations.

Acknowledgements. This work is supported by the NIH grant 1P01CA87634. 


\section{References}

1. H. Park, P. H. Bland, and C. R. Meyer, "Construction of an abdominal probabilistic atlas and its application in segmentation," IEEE Trans. Med. Imaging., vol. 22, pp. 483-492, 2003.

2. W. Toga and P. M. Thompson, "The role of image registration in brain mapping," Image and Vision Computing, vol. 19, pp. 3-24, 2001.

3. C. Studholme and V. Cardenas, "A template free approach to volumetric spatial normalization of brain anatomy," Pattern Recognition Letters, vol. 25, pp. 1191-1202, 2004.

4. S. Joshi, B. Davis, M. Jomier, and G. Gerig, "Unbiased diffeomorphic atlas construction for computational anatomy," NueroImage, vol. 23, pp. s151-s160, 2004.

5. D. L. G. Hill, P. G. Batchelor, M. Holden, and D. J. Hawkes, "Medical image registration," Physics in Medicine and Biology, vol. 46, pp. r1-r45, 2001.

6. C. Meyer, J. Boes, P. Bland, K. Zasandy, P. Kison, K. Koral, K. Frey, and R. Wahl, "Demonstration of accuracy and clinical versatility of mutual information for automatic multimodality image fusion using affine and thin plate spline warped geometric deformations," Medical Image Analysis, vol. 3, pp. 195-206, 1997.

7. F. L. Bookstein, "Principal warps: thin-plate splines and the decomposition of deformations," IEEE Trans. Pattern Anal. Mach. Intell., vol. 11, pp. 567-585, 1989.

8. F. W. Young and R. M. Hamer, Theory and application of Multidimensional sclaing: Eribaum Associates, 1994.

9. D. L. Collins, A. P. Zijdenbos, A. P. Kollokian, J. G. Sled, N. J. Kabani, C. J. Holmes, and A. C. Evans, "Design and Construction of a Realistic Digital Brain Phantom," IEEE Trans. Med. Imaging, vol. 17, pp. 463-468, 1998.

10. S. Marsland, C. J. Twining, and C. J. Taylor, "Groupwise non-rigid registration using polyharmonic clamped-plate splines," in MICCAI 2003, LNCS, vol. 2879, p.771-779, 2003.

11. G. E. Christensen and H. J. Johnson, "Consistent image registration," IEEE Trans. Med. Imaging., vol. 20, pp. 568-582, 2001. 